\title{
COMPARATIVE VISUALIZATION OF BIM GEOMETRY AND CORRESPONDING POINT CLOUDS
}

\author{
V. STOJANOVIC, R. RICHTER, J. DÖLLNER \& M. TRAPP \\ Computer Graphics Systems Chair, Hasso Plattner Institute, University of Potsdam, Germany
}

\begin{abstract}
We present a set of techniques for the combined and comparative visualization of 3D model geometry extracted from Building Information Models (BIM) and corresponding point clouds. It addresses the steady need to validate, update and combine BIM, in particular based on in-situ captured point clouds, throughout the whole lifecycle of buildings and facilities. To assess the present as-built interior and exterior in comparison to the as-designed or as-documented building representations, our techniques allow for deviation analysis and visualization, which serve as an effective method for enhancing stakeholder engagement. For example, Facility Management (FM) stakeholders can use deviation analysis and visualization to identify, inspect and monitor any spatial alterations both for interior and exterior building parts. Visualized instantaneous deviations can inform stakeholders of further need for investigation; they may not even have architecture, engineering and construction (AEC) expertise or access to BIM software. We describe a prototypical implementation that demonstrates the application of comparative deviation analysis and visualization. Finally, we discuss how the visualization output can provide a tool for a variety of stakeholders to improve applications and workflows for FM.
\end{abstract}

Keywords: 3D BIM, 3D Visualization, Deviation Analysis, Facility Management, Point Clouds

\section{INTRODUCTION}

To update and reference existing building features within BIM datasets, we need updating processes within the IT infrastructure supporting building operations and maintenance. This becomes most relevant if the whole lifecycle is addressed by IT solutions. Information sharing amongst stakeholders is a core requirement of BIM, including access to updated building documentation. For example, this is the case in the United Kingdom for all centrally procured government projects [1]. One effective way to inform FM stakeholders of changes within the current built environment is based on interactive visualization.

As a core function we can apply point cloud visualization for as-built representations. This is done to compare building interiors and exteriors with as-designed BIM Level-of-Detail (LOD) geometry models. Point clouds allow us to detect inconsistencies between the as-built and as-designed models, and this way they are key for all BIM update processes. Point clouds can also complement different BIM LOD geometry representations, for example, providing additional or missing details. LOD 3D geometries and associated location data can be extracted from BIM standard Industry Foundation Classes (IFC) files and used for deviation analysis. Built environment features can be captured with 3D scanning technologies such as Light Detection and Ranging (LiDAR) or computed by image-based reconstruction approaches. The acquired 3D point clouds represent the building as an unstructured and dense collection of discrete surface points [2]. In contrast, existing as-designed BIM 3D geometry data represents the building typically as a polygonal 3D model with attributes that refer to the semantics of building parts [3].

The approach for deviation analysis we investigate in this paper enables visual assessment of spatial differences between existing BIM geometry and captured point-cloud data, and allows us to identify and document new building features that are added in the post-construction phase (e.g. positioning of furniture, addition or alteration of wall segments). This paper addresses 
how deviation analysis can be visually communicated to various stakeholders for an initial assessment that complements the decision-making process. Our contributions in this paper include:

1. A method for extraction of BIM location data for alignment of point clouds within 3D geometry.

2. A real-time rendering technique for the combined, comparative visualization of BIM 3D geometry and point-cloud data.

3. A prototype software tool using 3D rendering techniques to display deviations between point-cloud data and BIM geometry.

4. Implementation hints for the deviation analysis visualization and a performance analysis.

\section{RELATED WORK}

BIM stakeholders required support for updating and referencing available building features with respect to existing or newly generated BIM. Taking into account the whole lifecycle, the operational cost of a building is generally far higher than the construction cost (five to seven times) and, therefore, the use of intuitive, transparent, and informative stakeholder engagement systems based on BIM provide benefits to operations, optimizations, and cost reduction [4].

According to Roper and Payant [5], the use of building automation within an existing IT infrastructure is the main cornerstone of an integrated workplace management system (IWMS). These IWMSs must be able to communicate and provide an informative analytical output of the state of a given FM operation to stakeholders. The two key features of using BIM in FM are information sharing between stakeholders and clash detection, which potentially can help reduce operational costs [6]. The combined use of data sharing principals provides further benefits that enable FM stakeholders from all other related FM practices to have access to critical building operation information [7].

Research conducted by Ebessen [8] shows that BIM is the leading IT type being currently investigated for use in FM applications. However, the main obstacle is being able to acquire and use the required data for decision making. Further, small to medium enterprises (SMEs) that are required to adopt BIM are confronted with high initial investment costs for the required BIM software, for example, due to increased training time and software license costs [9]. According to Kincaid [10], integrated FM has two key roles within an organization: (1) management of the organization and (2) management of the support and critical services of the organization. The areas of these operations are quite diverse in terms of complexity and their critical need for the operation of the facility. Design, planning and refurbishments are listed as the most sophisticated in terms of complexity and the most essential in terms of critical operation. Stakeholders who want to adopt BIM-based FM practices need to create a strategy that will help to reduce costs and redundancy of current FM systems in use and enable collaboration amongst concerned stakeholders who may come from different management backgrounds [11].

While traditional CAD-based software tools allow for more in-depth and broader exploration of building model data in $2 \mathrm{D}$ and $3 \mathrm{D}$, the use of such tools can be too complex and time consuming for stakeholders who do not have expertise is AEC domains to interpret the visual output from CAD-based software correctly [12]. Layouts of building spaces presented in 3D provide opportunities for FM personnel, for example, to obtain insights how features of a building are associated with facility use [13]. Lee et al. [14] describe the design, development 
and testing of a collaborative FM decision-making tool, using web-based interactive 3D visualization. Such viewing transformations may reveal elements that may otherwise be hidden, obscured or misinterpreted when read from a 2D floor plan [15]. Laing et al. [16] state that point clouds of built environments can capture a vast amount of information at very high resolutions, particularly in dense urban environments. The resulting massive point clouds provide insights into the state of the represented built environment for a given AEC or FM decision task. Unfortunately, as noted by Dimitrov and Golparvar-Fard [17] raw point clouds can contain a number of undesired artefacts, including: (1) noise introduced due to surface roughness, (2) undesired small objects, (3) occluding elements, (4) partially and/or incorrectly captured data and (5) increased point-cloud density due to overlapping scans.

Fadli et al. [18] noted that depending on the circumstances and application requirements, different resolutions and capture methods for point clouds can be used (e.g. combined photometry and laser-based scanning for cultural heritage visualization, and combined laser scanning and sensor data for emergency preparedness visualization. The use of point-cloud data alongside BIM has previously been treated as a separate process and mostly used for construction-related deviation analysis [19, 20]. A key feature of visual analysis focusing on spatial deviation is being able to examine point-cloud data sets of interior building features and compare them with existing BIM geometry data and associated semantics. This enables for initial visual assessment of any conflicting differences and also facilitates the documentation of new building features that are added in the post-construction phase [4, 21].

Research by Anil et al. [22] focused on using an existing commercial software tool to import as-is BIM data and captured point clouds to perform deviation analysis. This analysis focused on the accuracy of the reconstructed as-is BIM model data in comparison to the captured point clouds representing the actual built environment. The authors state the advantage of having access to immediately visualized results in $3 \mathrm{D}$, which can be inspected from any viewing angle. It indicates increased benefits in terms of costs and time to use point-cloud data to compare the accuracy of as-is BIM data rather than using physical measurement procedures. However, the authors note that the result of a deviation analysis visualization is subjective.

As an alternative to direct surface geometry based comparison methods, Kalasapudi et al. [23] recommend the use of a relational graph-based approach to measure deviations between as-designed BIM and as-is point-cloud data for internal mechanical, electrical and plumbing (MEP) components of a building. They propose a correlation matrix as a primary output, where each cell shows a comparison score based on the deviations from the as-designed BIM and as-is captured point-cloud data. The authors also adopted a reconstruction approach to comparing the as-is point-cloud data with the as-designed BIM geometry data. The 3D point cloud is segmented and reconstructed into primitive geometric shapes that are subsequently compared against corresponding as-designed BIM geometry. The resulting spatial relations are used to generate a relation-graph for the deviation analysis.

Combining geometry and point clouds in a single scene may lead to an overwhelming amount of information, for example, cluttered display. This can be remedied by applying the selective visualization process based on Shneiderman's Visual Information Seeking Mantra: Overview first, zoom and filter, then details on demand [24]. The use of geometry blending and interactive navigation to address occlusion and issues introduced by perspective projections are examples of the practical application of this mantra, and this has been discussed in work by Semmo et al. [25]. The initial deviation analysis visualization of combined BIM 3D geometry and point cloud data can inform the facility management personnel of operational features to be investigated further by other stakeholders. 


\section{VISUALIZATION OF POINT CLOUDS AND 3D GEOMETRY DATA}

This section presents the application of visualization methods for combined point cloud and geometry data presentation targeted towards stakeholder engagement. Specifically, it describes the application of visualization methods related to initial deviation analysis for FM. Figure 1 shows the deviation analysis prototypical tool in use.

\subsection{Visualization characteristics}

To visualize BIM 3D geometry and related point clouds, we have to understand where this visualization fits into the FM decision-making scope. Each level from decision making to stakeholder engagement (including visualization) can be thought of as a layer within a decisionmaking system associated with the FM stage of the building lifecycle process. Figure 2 illustrates the visualization process within the stakeholder engagement scope for FM

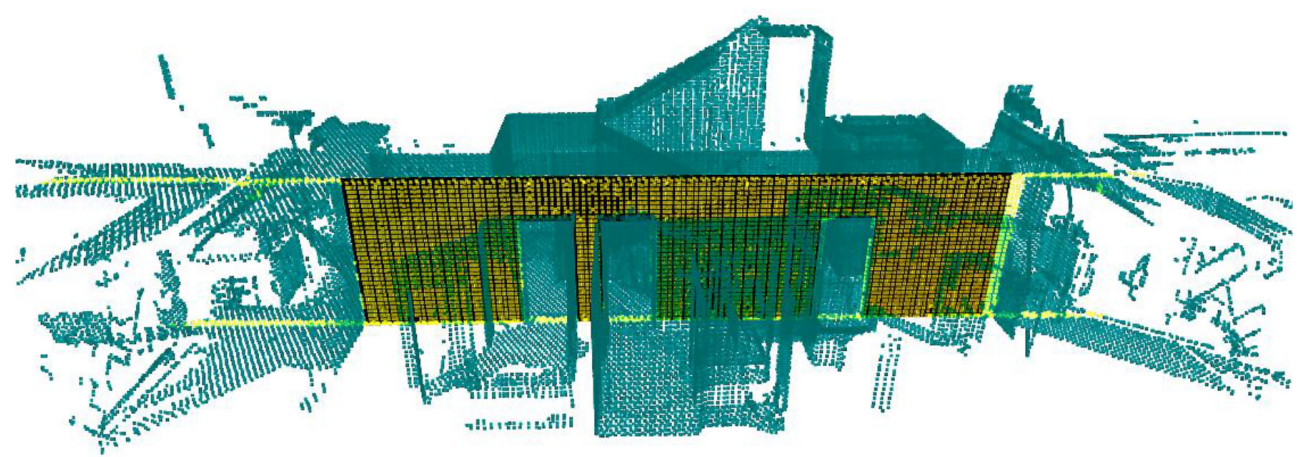

Figure 1: Visualization showing the deviations between a door frame and a wall segment. The yellow shaded points are within the accepted deviation range $($ e.g. $10 \mathrm{~cm}$ ).

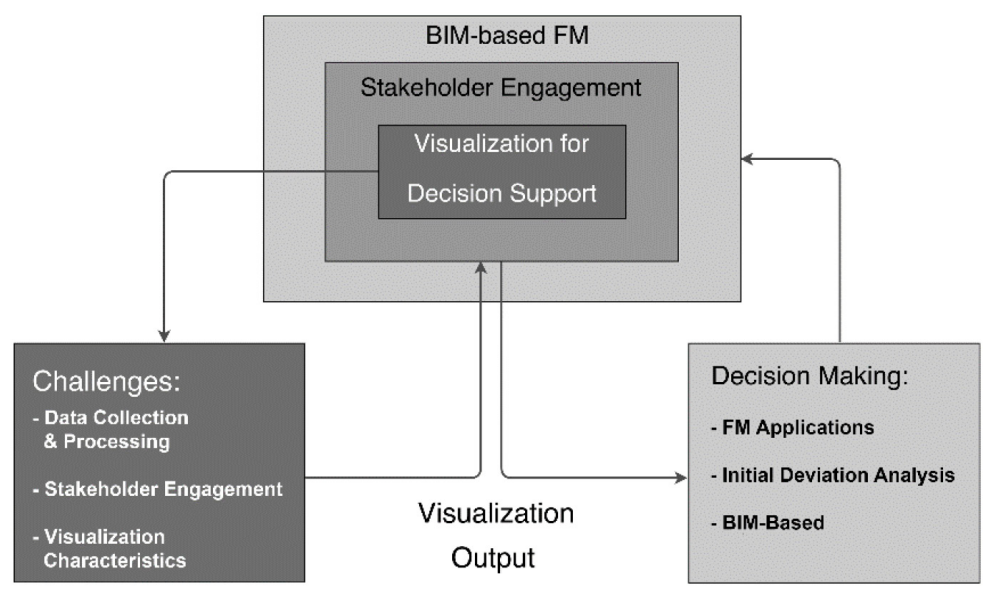

Figure 2: Illustration showing the scope of the visualization problem within FM applications. 
applications. The following components characterize the visualization of combined BIM, 3D geometry and related point clouds:

1. Geometry primitives: BIM 3D polygon data at specified LOD and point-cloud data.

2. 3D projection: These relate to scene presentation and user navigation (e.g. isometric vs. perspective projection).

3. Color encoding: Used to indicate areas-of-interest and key visual differences. They are also used as visual indicators based on the familiar built environment presentation.

4. Surface opacity: The surface opacity used to counterbalance the occlusion problem by setting a transparency value to the alpha (A) channel of the RGBA surface colour component of the geometry primitive types.

The values for these inputs can be specified either with user inputs, specific user actions or the default values defined by the BIM semantics. This enables a user driven interaction and inspection within the virtual 3D scene rendered in real-time. Additionally, Figure 3 illustrates complete high-level process flowchart for performing initial deviation analysis. The final output is intended to benefit the FM decision-making process.

\subsection{IFC Preprocessing and Point Cloud Alignment}

The 3D geometry data stored in IFC files are a major source for comparative representation. Since the geometric representations are stored as space partitions, a connectivity graph can be computed to generate a $3 \mathrm{D}$ volume of a building partition by linking all of the described nodes as edges $[9,26]$. Additional geometric representations such as curves, swept solids and constructive solid geometry (CSG) can also be used if available in the IFC dataset [27].

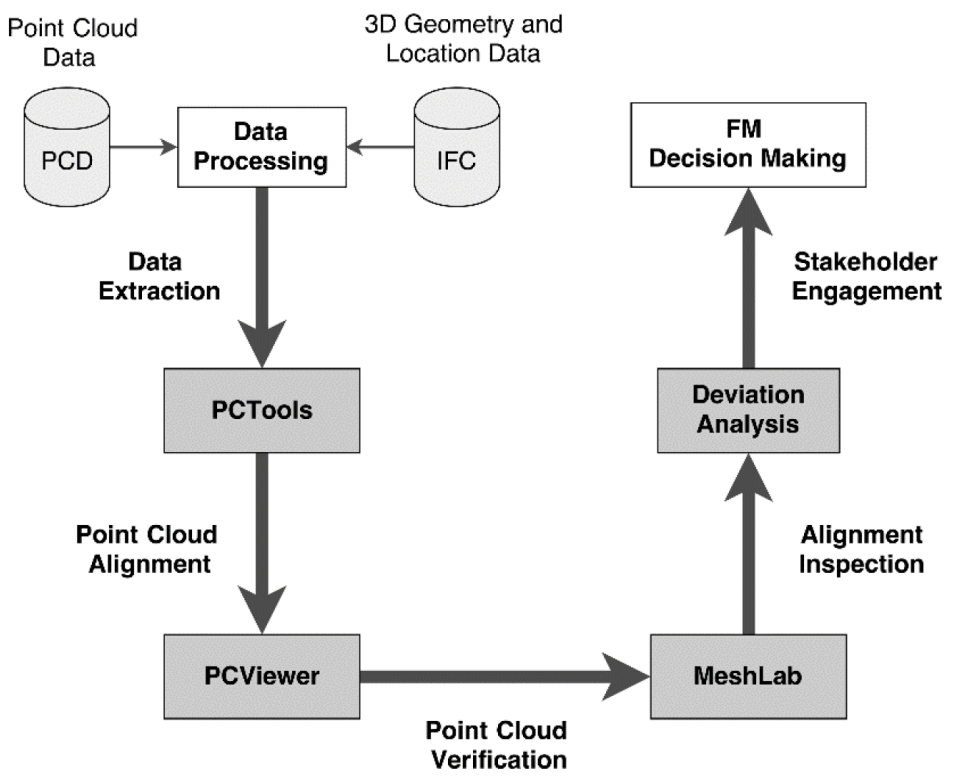

Figure 3: The process flowchart illustrating the pre-processing, and deviation analysis and visualization stages. 
Specified geometry segments are subsequently extracted at a desired LOD and required triangulation since the initial deviation analysis and rendering work with triangles as the primary 3D data type. These extracted geometry segments, along with their georeferenced locations, are used for deviation analysis; the extraction of location data from an IFC file is required to align the corresponding point cloud with the BIM 3D geometry. This step is optional if the point cloud and geometry data are already aligned. The alignment of the pointcloud data can include the transformation of separate or combined position, scaling and rotation computation.

Once the location data has been extracted from an IFC file, it can be applied to the point cloud. The transformations are applied as non-commutative matrix operations of separate or combined position, scaling and rotation computations. We used our own dedicated point cloud tool, PCTools, to perform the transformation operations on the corresponding point cloud, resulting in the correct alignment with the BIM geometry data used for the deviation analysis. The PCTool viewer used for visual inspection. PCTools is based on a framework is used for the out-of-core real-time visualization of massive 3D point clouds [28, 29]. PCTools was extended to include support for reading and applying IFC location semantics transformations to point cloud data. The parsing and extraction of the required IFC semantics was enabled by using the IFCOpenShell library. In the final pre-processing step after the transforms have been applied, the MeshLab software tool [30] was used to visually verify the transformed point cloud data with the extracted BIM geometry data. Figure 4 shows the process of the point-cloud data transformation. The software tools used for the pre-processing of the point clouds include the following: (1) PCTools, for point-cloud data alignment, (2) PCViewer, for optional point cloud visualization, (3) MeshLab, for optional combined point cloud and geometry visualization verification and (4) the prototype application for the initial deviation analysis visualization.

\subsection{Combined 3D geometry and point cloud visualization}

The next step after aligning the point cloud with the BIM geometry is to visualize the deviation values. This value will determine if the compared as-is point cloud data deviates spatially from the as-designed BIM geometry. This is accomplished using a combined 3D geometry and point cloud rendering approach.

The main objectives of the 3D rendering techniques include: (1) the 3D visualization of the built environment, (2) highlighting potential deviations between as-designed geometry and as-built point cloud data, (3) the presentation of building geometry that can differentiate between geometry surface features, (4) addressing the issue of occlusion by providing a transparency-based blending of both the geometry and point cloud data and (5) supporting the user to inspect the build environment representation in 3D in real-time using standard

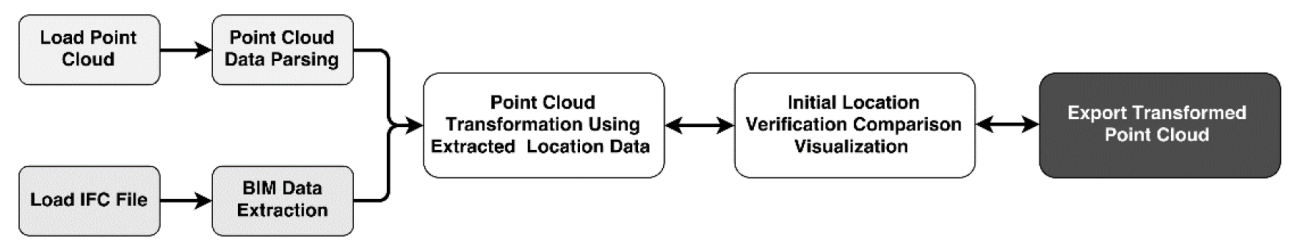

Figure 4: Point cloud transformation process flowchart. 


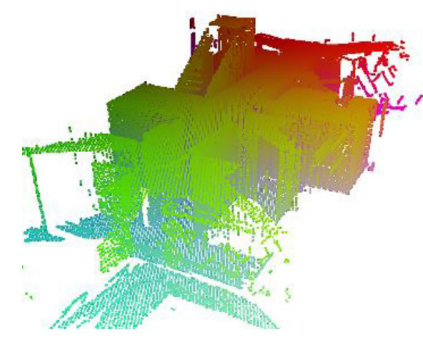

(a)

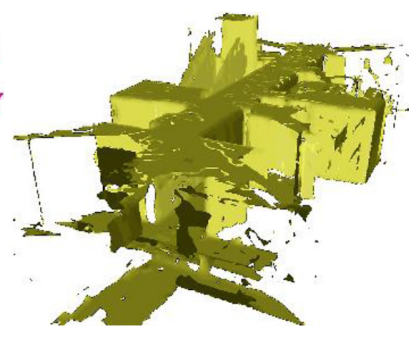

(b)

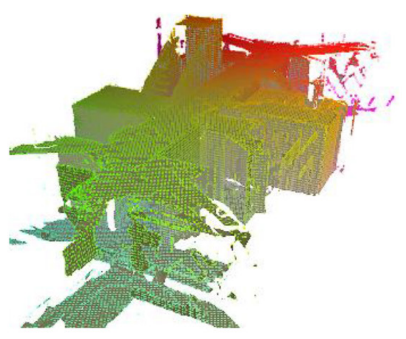

(c)

Figure 5: Methods used for the combined point cloud and BIM geometry data representation: (a) Point cloud rendering, (b) geometry and (c) blended point cloud over opaque geometry.

perspective projection. Figure 5 (a)-(c) shows each of the implemented rendering methods used for the combined rendering of point cloud and BIM geometry data.

\subsection{Point cloud deviation analysis visualization}

Two variants of deviation visualization are proposed: (1) binary distance colouring and (2) gradient distance colouring visualization styles. Figure 6 illustrates both different deviation analysis visualization methods. Given the pre-computed deviation factors, the deviation analysis visualization is implemented using a shader program. It basically maps the deviation factor either to a single colour or a gradient. The binary approach uses a threshold value to determine at what distance the points are deviating. This value can be adjusted by the user interactively using a graphical user interface. This allows the user to set an acceptable 'fault tolerance' for the comparison of as-designed versus as-is built of the geometry primitive types. For example, the binary visualization style shades all points beyond a threshold level as red and all points within the threshold value as blue. The gradient visualization method allows for continuous shading of points from those smaller than the threshold to those greater by linearly interpolating

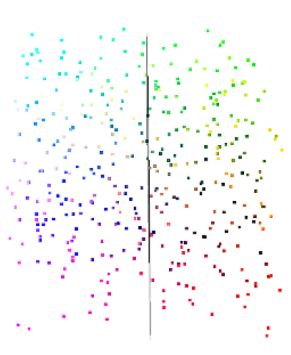

(a)

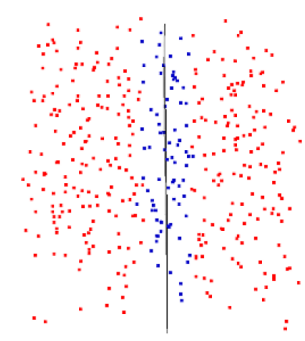

(b)

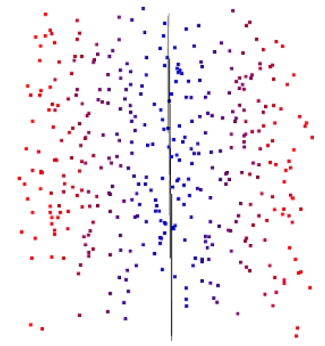

(c)

Figure 6: Exemplary comparison between (a) normal point cloud colouring, (b) binary distance and (c) gradient distance colouring visualization styles. 
between two different colours. The interpolation factor is based on the distance value of points from the plane of the BIM geometry.

\section{RESULTS AND DISCUSSION}

The test case for the implemented visualization methods include an indoor point cloud (provided by SAP Innovation Center in Potsdam, Germany). The deviation geometry model was intentionally offset by the authors, by approximately $40 \mathrm{~cm}$ from the correct as-designed BIM geometry model. This shows the clear misalignment of the door frames in comparison to the correct geometry test model. The aligned wall model versions of both the correct and deviated geometries were exported as OBJ model files, which were then first verified in MeshLab for correct alignment with the point cloud.

\subsection{Deviation analysis visualization results}

In the test case, an assumption of using segmented BIM geometry in the pre-processing step was made. Figure 7(a)-(c) shows the extracted BIM geometry models that were used. The result images in Figure 8(a)-(d) show the original and altered indoor geometry models used in the deviation analysis visualization. The visualized deviation levels are computed using a

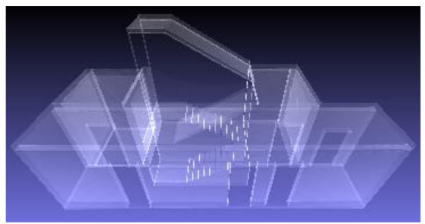

(a)

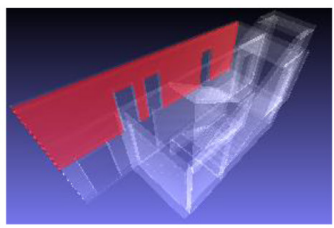

(b)

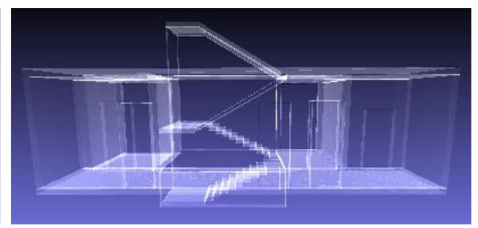

(c)

Figure 7: Images (a)- (c) showing the extracted BIM geometry from where the wall segment was used.

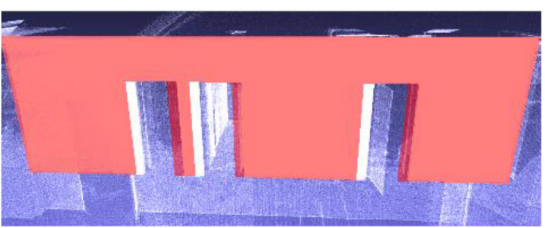

(a)

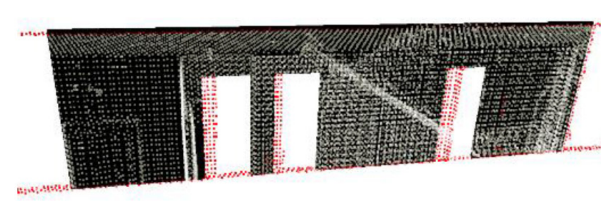

(c)

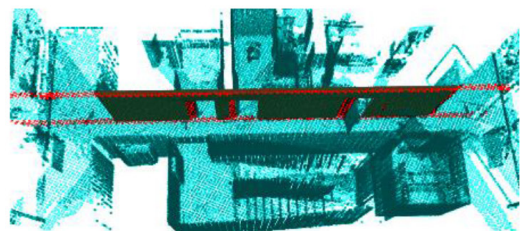

(b)

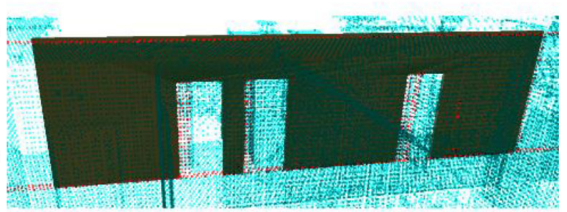

(d)

Figure 8: Sequence of images (a)-(d) showing the deviation analysis visualization. 


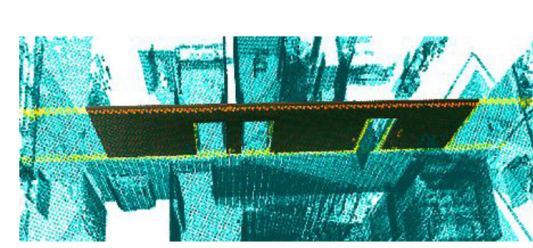

(a)

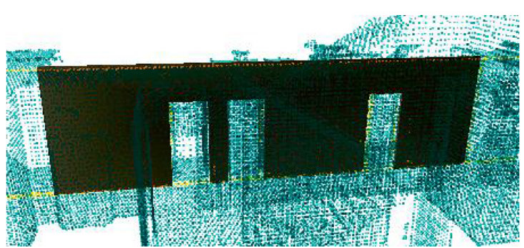

(b)

Figure 9: Images (a) and (b) shows the top-down and front view of the correct wall alignment.

threshold value and can be assigned to a required unit (e.g. millimetres, centimetres and meters), depending on the comparison scenario requirements. Figure 9(a)-(b) shows how the loaded geometry is presented within the correct alignment space alongside the point-cloud model. The results show that the $3 \mathrm{D}$ inspection of the deviation analysis can be performed in real-time using as-designed BIM and as-is point-cloud data. The visualization outputs can viewed and inspected from any angle in 3D space and the problem of occlusion is approached using blending methods.

\subsection{Technical implementation details}

The main visualization component of the prototype application is implemented in $\mathrm{C}++$ using the $Q t 3 D$, which is part of the $Q t 5.7$ framework. $Q t 3 D$ is a high-level wrapper for OpenGL that is based on a combined scenegraph and framegraph rendering system. It is based on the concept of a scenegraph that describes how the scene is rendered and stores each object in the scene as a component in a node-based hierarchical tree structure to accelerate real-time rendering operations [31]. The main advantage of using a framegraph is being able to configure low-level 3D rendering states using high-level function calls. Both the point cloud and triangulated BIM geometry primitives are rendered using custom rendering methods implemented as shaders and executed on the Graphics Processing Unit (GPU) using the programmable graphics pipeline [32]. The shader rendering methods are used to adjust the transparency values of the points and the surface shading within the framegraph system. The loading of the point clouds in the prototype application was implementing using the Point Cloud Library [33].

\subsection{Performance evaluation and discussion}

The original point-cloud data has $1,053,735$ points. Additionally, a lower resolution point cloud consisting of 63,618 points was generated based on the original (resampled by a factor of 0.15 ). This was done to mimic different point cloud scanning resolutions that may be expected from typical scanning devices. The evaluation is performed on a commodity desktop PC with an Intel Core i5-6500 CPU at 3.2GHz, 8GB RAM and an NVIDIA GeForce GT 630 graphics card with $2 \mathrm{~GB}$ of dedicated memory. The prototypical implementation runs in real-time with approx. 60 frames-per-second with both the combined low-density point cloud, and the combined high-density point and BIM geometry.

The performance of the pre-computation algorithm that maps the distance values of the points to the segmented BIM geometry runs in linear time (running in $O(n * k)$ time - where $n$ is the number of points and $k$ is the number segmented BIM geometry objects). The 
pre-computation algorithm creates a plane object around the segmented BIM geometry data. It then checks how far each point is from the plane and maps the distance of the points based on the input threshold level. This means that the time taken to compute the distance values compares linearly to the number of input objects. Once the distance values have been computed they are stored in a new point cloud file, so re-computation is not required for future use if the same point cloud and geometry models are compared. A remedy to increase the pre-computation performance is to use a dynamic data structure such as an octree portioning method. In this representation, each octree node contains a minimum of 1 point, which can be compared to any BIM geometry using raycasting intersection tests. This feature is implemented in the PCR framework for use with larger and more complex point-cloud data.

\subsection{Future work}

The error of alignment between the point cloud and 3D geometry data was not taken into account at this stage of our research, but this will be investigated in the future. It is assumed that the point cloud is aligned based on the extracted IFC model location data, and that it is accurate to a visual degree of acceptability for initial deviation analysis visualization output. There are also plans to test the application with actual stakeholders involved in FM and BIM, in order to determine how beneficial its use would be.

\section{CONCLUSIONS}

Point clouds can be considered as key elements for an approach towards image-based 4D BIMs. In a sense that they can be acquired by image-based technology and allow for updating BIMs over time. Point clouds can be used to capture the state of the built environment quickly and at low costs, and they are not restricted and do not assume specific types of geometry or topology due to their generality. This way they provide a constant source of spatial information that facilitates generating and updating of BIMs. There are many uses of point clouds for AEC and FM applications - one is the deviation analysis and visualization described here. The key advantage of using point clouds for deviation analysis and visualization is that they directly provide added values to all the stakeholders. The use of point clouds alongside BIM also opens the path towards more sophisticated applications, for example, interior catalogues of office spaces or as-is BIM generation and reconstruction applications. Finally, integrating up-to-date point clouds and BIMs is crucial for Industry 4.0 applications, for example, based on sensor data.

\section{ACKNOWLEDGEMENTS}

The authors would like to thank Martin Fischer and Jan van Dieken for their implementation contributions. This work was partially funded by the Federal Ministry of Education and Research (BMBF), Germany, within the InnoProfile Transfer research group '4DnD-Vis' (www.4dndvis.de), the Research School on 'Service-Oriented Systems Engineering' of the Hasso Plattner Institute and SAP Innovation Center in Potsdam, Germany.

\section{REFERENCES}

[1] Gledson, B., Greenwood, D., Routledge, P., Watson, R. \& Woddy, P., Preparing to work in level 2 BIM: an innovative approach to a training and project-based learning, 2016.

[2] Levoy, M. \& Whitted, T. The use of points as a display primitive. University of North Carolina, Department of Computer Science, 1985. 
[3] Tang, P., Huber, D., Akinci, B., Lipman, R. \& Lytle, A. Automatic reconstruction of as-built building information models from laser-scanned point clouds: A review of related techniques. Automation in Construction, 19(7), pp. 829-843, 2010. https://doi.org/10.1016/j.autcon.2010.06.007

[4] Lee, S.K., An, H.K. \& Yu, J.H., An extension of the technology acceptance model for BIM-based FM. In Construction Research Congress 2012: Construction Challenges in a Flat World, pp. 602-611, 2012.

[5] Roper, O.K. \& Payant, P.K., The facility management handbook. AMACOM, 2009.

[6] Eastman, C.M., Teicholz, P., Sacks, R. \& Liston, K., BIM handbook: A guide to building information modeling for owners, managers, designers, engineers and contractors. In BIM Handbook, John Wiley \& Sons, Hoboken, New Jersey, pp. 170-171, 2011.

[7] Kensek, K., BIM guidelines inform facilities management databases: a case study over time. Buildings, 5(3), pp. 899-916, 2015. https://doi.org/10.3390/buildings5030899

[8] Ebbesen, P., Information technology in facilities management-a literature review. EuroFM, (1.4), 2015.

[9] Muñoz, V. \& Arayici, Y., Using free tools to support the BIM coordination process into SMEs. Building Information Modelling (BIM) in Design, Construction and Operations, 149, pp. 33-41, 2015.

[10] Kincaid, D., Integrated facility management. Facilities, 12(8), pp. 20-23, 1994. https://doi.org/10.1108/02632779410062353

[11] Ibrahim, K.F., Abanda, F.H., Vidalakis, C. \& Woods, G., BIM for FM: input versus output data, 2016.

[12] Woo, J. H. BIM (building information modeling) and pedagogical challenges. In Proceedings of the 43rd ASC National Annual Conference, pp. 12-14, 2006.

[13] Fischer, M., Haymaker, J. \& Liston, K., Benefits of $3 D$ and 4D models for facility managers and AEC service providers. 4D CAD and visualization in construction developments and applications, pp. 1-32, 2003.

[14] Lee, W.L., Tsai, M.H., Yang, C.H., Juang, J.R. \& Su, J.Y., V3DM+: BIM interactive collaboration system for facility management. Visualization in Engineering, 4(1), 2016. https://doi.org/10.1186/s40327-016-0035-9

[15] Atazadeh, B., Kalantari, M., Rajabifard, A., Ho, S., \& Champion, T., Extending a BIMbased data model to support 3D digital management of complex ownership spaces. IJGIS, pp. 1-24, 2016.

[16] Laing, R., Leon, M., Isaacs, J. \& Georgiev, D. Scan to BIM: the development of a clear workflow for the incorporation of point clouds within a BIM environment. WIT Transactions on the Built Environment, 149, pp. 279-289, 2015. https://doi.org/10.2495/BIM150241

[17] Dimitrov, A. \& Golparvar-Fard, M., Segmentation of building point cloud models including detailed architectural/structural features and MEP systems. Automation in Construction, 51, pp. 32-45, 2015.

https://doi.org/10.1016/j.autcon.2014.12.015

[18] Fadli, F., Barki, H., Boguslawski, P. \& Mahdjoubi, L., 3D scene capture: a comprehensive review of techniques and tools for efficient Life Cycle Analysis (LCA) and Emergency Preparedness (EP) applications. WIT Transactions on the Built Environment, 149, pp. 85-96, 2015. 
[19] Qu, T., \& Sun, W., Usage of 3D point cloud data in BIM (Building Information Modelling): Current Applications and Challenges, 2015.

[20] Tuttas, S., Braun, A., Borrmann, A. \& Stilla, U., Acquisition and consecutive registration of photogrammetric point clouds for construction progress monitoring using a 4D BIM. $P F G, \mathbf{8 5}(1)$, pp. 3-15, 2017.

[21] Barki, H., Fadli, F., Shaat, A., Boguslawski, P. \& Mahdjoubi, L., BIM models generation from 2D CAD drawings and 3D scans: an analysis of challenges and opportunities for AEC practitioners. Building Information Modelling (BIM) in Design, Construction and Operations, 149, pp. 369-380, 2015.

[22] Anil, E.B., Tang, P., Akinci, B. \& Huber, D. Assessment of quality of as-is building information models generated from point clouds using deviation analysis. In Proceedings of SPIE, 2011.

[23] Kalasapudi, V.S., Turkan, Y. \& Tang, P., Toward automated spatial change analysis of MEP components using 3D point clouds and as-designed BIM models. In 3DV (Vol. 2, pp. 145-152). IEEE, 2014.

[24] Shneiderman, B., The eyes have it: A task by data type taxonomy for information visualizations. In Visual Languages, 1996. Proceedings., IEEE Symposium, pp. 336-343, 1996.

[25] Semmo, A., Trapp, M., Kyprianidis, J. E. \& Döllner, J., Interactive visualization of generalized virtual 3D city models using level-of-abstraction transitions. Computer Graphics Forum 2012, 31(3), pp. 885-894, 2012. https://doi.org/10.1111/j.1467-8659.2012.03081.x

[26] Diakité, A.A. \& Zlatanova, S., Valid space description in BIM for 3D Indoor. IJ3DIM, 5(3), pp. 1-17, 2016. https://doi.org/10.4018/ij3dim.2016070101

[27] BUILDINGSMART. IfcShapeRepresentation, 2017, available at: https://tinyurl.com/ k9qk77m. (accessed 29 March, 2017).

[28] Richter, R., Discher, S. \& Döllner, J., Out-of-core visualization of classified 3d point clouds. 3D Geoinformation Science, 227-242, 2015. https://doi.org/10.1007/978-3-319-12181-9_14

[29] Richter, R., Kyprianidis, J. E. \& Döllner, J., Out-of-Core GPU-based change detection in massive 3D point clouds. Transactions in GIS, 17, pp. 724-741, 2013. https://doi.org/10.1111/j.1467-9671.2012.01362.x

[30] Cignoni, P., Corsini, M. \& Ranzuglia, G., Meshlab: an open-source 3d mesh processing system. Ercim News, 73(45-46), p. 6, 2008.

[31] Lamire, P. Qt3D 2.0: The FrameGraph. KDAB, 2015, available at: https://tinyurl.com/ $\lg 6$ n2fs. (accessed 13 February, 2017).

[32] Akenine-Möller, T., Haines, E. \& Hoffman, N., Real-time rendering. CRC Press, 2008.

[33] Rusu, R. B. \& Cousins, S. 3D is here: Point Cloud Library (PCL). IEEE ICRA, 2011. 